\title{
Clinical utility gene card for: Hypophosphatasia - update 2013
}

\author{
Etienne Mornet ${ }^{\star, 1,2}$, Christine Hofmann ${ }^{3}$, Agnès Bloch-Zupan ${ }^{4,5,6}$, Hermann Girschick $^{7}$ and \\ Martine Le Merrer ${ }^{8}$
}

European Journal of Human Genetics (2014) 22, doi:10.1038/ejhg.2013.177; published online 7 August 2013

Update to: European Journal of Human Genetics (2010) 19, doi:10.1038/ejhg.2010.170; published online 27 October 2010

\section{DISEASE CHARACTERISTICS}

1.1 Name of the disease (synonyms)

Hypophosphatasia (HP), HPP, rathbun disease and phosphoethanolaminuria.

\subsection{OMIM\# of the disease \\ 146300, 241500 and 241510}

1.3 Name of the analysed genes or DNA/chromosome segments Alkaline phosphatase (AP) liver type (ALPL), 1p36.1-p34.

Used other names: TNAP and tissue nonspecific AP.

\subsection{OMIM\# of the gene(s)}

171760.

\subsection{Mutational spectrum}

Over 250 different disease-causing mutations have been reported in the ALPL gene mutations.

Database http://www.sesep.uvsq.fr/03_hypo_mutations.php. The distribution is as follows: $75.5 \%$ missense mutations; $10.5 \%$ small deletions; $6 \%$ splicing mutations; $4 \%$ nonsense mutations; $2 \%$ small insertions; and $1 \%$ or less: complex insertion/deletions, large deletions and mutations in the regulatory sequence. The large proportion of missense mutations with various effects on the enzymatic activity of AP, based upon in vitro studies, has been correlated with the high clinical variability. ${ }^{1}$ A number of missense mutations exhibit a dominant-negative effect $^{2-5}$ explaining dominant inheritance of mild forms of the disease. ${ }^{6-8}$

\subsection{Analytical methods}

The main strategy for mutation screening consists in sequencing of genomic exonic DNA, including flanking intronic sequences. This allows the detection of approximately 95\% of mutations in patients with HP. The analysis may be completed by screening for large deletions by quantitative multiplex PCR of short fragments, ${ }^{9}$ but this does not significantly increase the detection rate because large deletions seem rare in the ALPL gene. For prenatal diagnosis, a set of linked microsatellites sequences strongly linked to the ALPL gene may be used for indirect diagnosis and/or excluding maternal cell contamination.

\subsection{Analytical validation}

The existence of mutations is confirmed by testing the parental DNA or, when the parental DNA is not available, by sequencing a second and independant PCR product. Newly discovered missense mutations may be tested in vitro by site-directed mutagenesis for enzymatic activity, protein stability, cell localisation and so on, clarifying their disease-causing role and estimating their degree of severity. ${ }^{1}$

1.8 Estimated frequency of the disease (incidence at birth ('birth prevalence') or population prevalence)

According to a report from 1957 in Toronto, Canada, ${ }^{10}$ the incidence of severe cases of HP (perinatal and infantile forms) is 1 in 100000 . On the basis of molecular diagnosis in France and Europe, the incidence of severe forms has been estimated at $1 / 300000$, a lower value that may reflect a founder effect previously observed in the region of Toronto. ${ }^{11}$ Study of the prevalence of mild forms (prenatal benign, childhood, adult and odontoHP) has not been reported yet, but it is expected higher because of low selective pressure and because heterozygotes for some mutations may express the disease.

1.9 If applicable, prevalence in the ethnic group of investigated person

Greenberg et al. ${ }^{12}$ suggested that the disease prevalence in Canadian Mennonites could be up to 1 in 2500 . HP seems very rare in populations of black ancestry. ${ }^{13}$

${ }^{1}$ Unité de Pathologie Cellulaire et Génétique UPRES-EA2493, Faculté de Médecine Paris Ile de France Ouest PRES Universud Paris, University of Versailles Saint Quentin en Yvelines, Versailles, France; ${ }^{2}$ Unité de Génétique Constitutionnelle, Centre Hospitalier de Versailles, Paris, France; ${ }^{3}$ Funktionsbereich Pädiatrische Rheumatologie, Immunologie und Osteologie, Kinderklinik und Poliklinik Universität Würzburg, Würzburg, Germany; ${ }^{4}$ Faculty of Dentistry, University of Strasbourg, Strasbourg, France; ${ }^{5}$ Reference Centre for Orodental Manifestations of Rare Diseases, Pôle de Médecine et Chirurgie Bucco-Dentaires, Hôpitaux Universitaires de Strasbourg, Strasbourg, France; 6 Institute of Genetics and Molecular and Cellular Biology (IGBMC), Inserm U964, CNRS-UdS UMR7104, Illkirch, France; ${ }^{7}$ Vivantes Klinikum im Friedrichshain, Department of Pediatrics, Clinic for Pediatric and Adolescent Medicine, Berlin, Germany and ${ }^{8}$ U781 et Département de Génétique, Centre de Référence des Maladies Osseuses Constitutionnelles, Hopital Necker-Enfant Malades, Paris, France

*Correspondence: Dr E Mornet, Unité de Génétique Constitutionnelle, Centre Hospitalier de Versailles, 2 rue Jean-Louis Forain, Le Chesnay 78150 , France. Tel: + 33139638013 ; Fax: + 331396380 12; E-mail: etienne.mornet@uvsq.fr 


\subsection{Diagnostic setting}

\begin{tabular}{llc} 
& Yes & No \\
A. (Differential) diagnostics & $\bigotimes$ & $\square$ \\
B. Predictive Testing & $\bigotimes$ & $\square$ \\
C. Risk assessment in relatives & $\bigotimes$ & $\square$ \\
D. Prenatal & $\bigotimes$ & $\square$ \\
\hline
\end{tabular}

Comment:

Not applicable.

\section{TEST CHARACTERISTICS}

\begin{tabular}{|c|c|c|c|c|}
\hline & \multicolumn{2}{|c|}{ Genotype or disease } & \multirow{2}{*}{$\begin{array}{l}\text { A: True positives } \\
\text { B: False positives }\end{array}$} & \multirow{2}{*}{$\begin{array}{l}\text { C: False negative } \\
\text { D: True negative }\end{array}$} \\
\hline & Present & Absent & & \\
\hline \multicolumn{5}{|l|}{ Test } \\
\hline Positive & A & B & $\begin{array}{l}\text { Sensitivity: } \\
\text { Specificity: }\end{array}$ & $\begin{array}{l}A /(A+C) \\
D /(D+B)\end{array}$ \\
\hline Negative & C & D & $\begin{array}{l}\text { Positive predictive value: } \\
\text { Negative predictive value: }\end{array}$ & $\begin{array}{l}A /(A+B) \\
D /(C+D)\end{array}$ \\
\hline
\end{tabular}

\subsection{Analytical sensitivity \\ (proportion of positive tests if the genotype is present) \\ Nearly $95 \%$.}

\subsection{Analytical specificity \\ (proportion of negative tests if the genotype is not present) \\ Nearly $100 \%$.}

\subsection{Clinical sensitivity \\ (proportion of positive tests if the disease is present)}

The clinical sensitivity can be dependent on variable factors such as age or family history. In such cases, a general statement should be given, even if a quantification can only be made case by case.

Nearly $95 \%$.

\subsection{Clinical specificity}

(proportion of negative tests if the disease is not present)

The clinical specificity can be dependent on variable factors such as age or family history. In such cases, a general statement should be given, even if a quantification can only be made case by case.

Nearly $100 \%$.

\subsection{Positive clinical predictive value}

(life-time risk to develop the disease if the test is positive)

$100 \%$ in recessive inheritance.

Up to $30-40 \%$ in mild forms with dominant inheritance, depending on the mutation.

\subsection{Negative clinical predictive value}

(probability not to developing the disease if the test is negative)

Assume an increased risk based on family history for a non-affected person. Allelic and locus heterogeneity may need to be considered.

Index case in that family had been tested: $100 \%$.

Index case in that family had not been tested: $95 \%$.

\section{CLINICAL UTILITY}

3.1 (Differential) diagnostics: The tested person is clinically affected (To be answered if in 1.10 'A' was marked)

(1) Diagnosis: based on clinical courses and severity, HP has been divided into six major subtypes with different prognoses. The perinatal form is the most severe one. It results in stillbirth or death a few days after birth due to hypoplastic lungs, difficulty to treat seizures, extensive hypomineralisation, deformities of bone and disturbances of the $\mathrm{Ca} / \mathrm{P}$ metabolism. In the prenatal benign form, despite prenatal findings on fetal ultrasound, there is a spontaneous improvement of skeletal defects. The patients manifest limb shortening and bowing and often dimples overlaying the long bones anomalies, and ultrasounds reveal progressive improvement of the skeletal anomalies and mineralisation during the third trimester of the pregnancy and after birth. ${ }^{14-16}$ Clinical signs of the infantile form appear during the first 6 months of life including rickets, premature craniosynostosis, irritability, seizures and nephrocalcinosis due to hypercalciuria. Later, premature loss of deciduous teeth is common. Death within the first year of life is common, but the precise risk is not understood. The childhood form in most cases presents after the first 6 months of life and is characterised by rickets causing a short stature, delayed walking and a waddling gait due to bone deformities and pain of the lower extremities. Later, premature loss of teeth often leads to diagnosis. It occurs before 5 years of age, and teeth are generally lost with intact root. Adult HP presents with osteomalacia, chondrocalcinosis, osteoarthropathy and stress fractures during middle age in patients who often had a history of mild rickets in childhood. Many patients present loss of permanent teeth. Odontohypophosphatasia is characterised by premature exfoliation of primary and/or permanent teeth and/or severe dental caries, not associated with abnormalities of the skeletal system. However, it should be noticed that the disease spectrum is a continuum, and that these six clinical subtypes can overlap significantly; for example, patients with adult HP often had musculoskeletal symptoms already in childhood. The infantile and the childhood form might be difficult to distinguish, because early symptoms might be present in the first months of life in both subtypes. In addition, dental abnormalities are frequent in other forms of HP. According to the severity of the disorder, some dental defects were infrequent, whereas other are always present. ${ }^{17}$ They consist of abnormal tooth shape (small bulbous crown, cervical constrictions and enlarged pulp spaces), abnormal tooth structure (enamel, dentin and cementum formation), tooth colour, dental anomalies of tooth eruption/exfoliation with premature loss of predominantly the primary and also the permanent dentition. Delayed eruption of teeth and primary teeth impaction (ankylosis) are also recorded.

(2) Differential diagnosis: The differential diagnosis of HP depends on the age at which the diagnosis is considered.

In utero: osteogenesis imperfecta (OI) type II, campomelic dysplasia and chondrodysplasias with bone mineralisation defect

At birth: outwardly difficult to distinguish, radiographs readily distinguish OI (type II) campomelic dysplasia and chondrodysplasias with bone mineralisation defect, from HP.

Infancy and childhood: inborn errors of energy metabolism, organic acidemia, primary and secondary rickets, neglect and nonaccidental trauma.

- Nutritional and/or vitamin D deficiency, vitamin D resistance or renal osteodystrophy

- OI (typically type III in infancy or type IV later on)

- Cleidocranial dysostosis (OMIM 119600) 
- Cole-Carpenter syndrome (OMIM 112240)

- Hadju-Cheney syndrome (OMIM 102500)

- Chronic recurrent multifocal osteomyelitis (OMIM 259680) $)^{18}$

- Idiopathic juvenile osteoporosis (OMIM 259750)

- Renal osteodystrophy

- Adult and odontoHP

- Osteoarthritis and pseudogout

- Osteopenia/osteoporosis

- Diseases with paraspinal ligament ossification (Forestier disease, arthropathy with calcium deposition)

- Premature exfoliation of teeth can occur in a context of periodontal disease, as part of a connective tissue disorder such as EhlersDanlos syndrome (130050 type IV; 130080 type VIII), or associated with neutropenia, such as ELA2-related neutropenia (OMIM 202700), Papillon-Lefevre syndrome (OMIM 245000) and Haim-Munk syndrome (HMS OMIM 245010), Chediak-Higashi syndrome (OMIM 214500) or as Agressive periodontitis (OMIM 170650). However, in HP, premature loss of primary and eventually then permanent teeth occurs without root resorption (fully rooted teeth) and without inflammation of the gingivae and periodontium and shows on radiograph alveolar bone loss.

- Dentin dysplasia type I (OMIM \%125400)

\subsubsection{Can a diagnosis be made other than through a genetic test?}

\begin{tabular}{|c|c|c|}
\hline No & $\square$ (continue with 3.1 .4 ) & \\
\hline \multirow[t]{7}{*}{ Yes } & $\nabla$ & \\
\hline & Clinically & $凶$ \\
\hline & Imaging & $凶$ \\
\hline & Endoscopy & $\square$ \\
\hline & Biochemistry & 凶 \\
\hline & Electrophysiology & $凶$ \\
\hline & Other (please describe) & \\
\hline
\end{tabular}

\subsubsection{Describe the burden of alternative diagnostic methods to the patient}

Diagnosis of HP often can cause considerable problems. On one hand, it is a rare disease with a variety of differential diagnoses. On the other hand, different HP subtypes present a partly comparable spectrum of symptoms. Conventional alternative diagnosis is based on laboratory assays and radiographic imaging and may be confirmed by genetic testing. Total serum AP activity is below the age-related normal range. AP activity depends on age, sex and on laboratory procedures. However, a reduced AP activity is only a helpful diagnostic indicator but it is not HP specific. Other conditions may also show reduced levels of AP including early pregnancy, hypothyroidism, anaemia, coeliac disease or zinc deficiency. In general, residual serum AP activity has directly been linked to disease severity. Patients with perinatal forms often have a total serum AP $<20 \%$ of the normal range, whereas a milder form (infantile or childhood form) has to be considered, if AP values are clearly below the lower limit. Patients with odontoHP and some with a mild end of the childhood HP spectrum have values slightly below the lower limit. Patients with singular heterozygous mutations or autosomaldominant inheritance often exhibit a considerable residual AP activity. Separation of an autosomal dominant with a compound heterozygous disease state on the basis of the biochemical AP activity, however, is usually impossible.

Reduced enzyme activity results in accumulation of its substrates including pyridoxal-5'-phosphate, inorganic pyrophosphate and phosphoethanolamine, which can be detected in serum and urine. Often serum calcium and phosphate are normal or slightly increased. Urinary calcium excretion might be above the normal range. Careful surveillance including ophthalmological and neurological examination is recommended in young patients with newly diagnosed craniosynostosis, perhaps complimented by an invasive epidural monitoring of intracranial pressure.

Sequencing of the ALPL gene is essential to confirm the diagnosis of HP when biochemical and clinical data are not clear enough or in the prenatal assessment of severe HP in couples with a previously affected child or pregnancy. Genetic consultation is recommended before genetic testing is done.

\subsubsection{How is the cost effectiveness of alternative diagnostic methods to be judged?}

Biochemical testing does not exclude genetic testing and vice versa. Both diagnostic procedures add to the clinical picture and to aspects of severity and complications in the follow-up. Biochemical testing is inexpensive and may be measured in any routine laboratory. However, in order to avoid diagnosis pitfalls, appropriate collection tubes (serum separator tubes) and correct reference range matched for sex and age must be used.

\subsubsection{Will disease management be influenced by the result of a} genetic test?

No $\square$

Yes $\otimes$

Therapy Treatment with biphosphonates has been reported

(please describe) disappointing ${ }^{19}$ and even detrimental, ${ }^{20}$ whereas teriparatide (the recombinant human parathyroid hormone PTH 1-34) was successfully used to improve and resolve metatarsal stress fractures in adult HP, however, depending on the number of mutations and on the nature of the mutation. ${ }^{21-24}$ Nonsteroidal anti-inflammatory drugs (NSAIDs) have been shown to provide clinical improvement with reduced pain, increased muscle strength and a normalised walking distance ${ }^{25,26}$ (see also management section).

Enzyme replacement therapy (ERT) has been investigated and successively tested in mice. ${ }^{27}$ Clinical trials in infants and young children with severe HP showed very encouraging results, ${ }^{28}$ and ERT could be available soon for HP patients. Genetic testing can confirm the clinical/biochemical diagnosis but should not delay starting treatment in severe forms.

Other therapeutic approaches are in development, including a gene therapy vector that shows promising results in a mouse model of hypophophatasia. ${ }^{29}$

Prognosis The prognosis depends on clinical course and severity.

(please describe) The most severe types of HP, that is, perinatal and infantile forms, are often diagnosed on clinical and radiological criteria, and genetic testing confirms the diagnosis and is helpful for genetic counselling. However, childhood and adult forms, as well as odontoHP, are more difficult to diagnose on clinical, radiological and biochemical criteria, and clinicians having uncertainties may request genetic testing to argue for the diagnosis. The perinatal form is almost always lethal within days or weeks. However, in the rare prenatal benign form, there is a progressive 
improvement of skeletal defects during the third trimester of the pregnancy, and patients finally are affected with mild $\mathrm{HP}^{14,15}$ or with more severe, non-lethal, however, clinical forms of HP. ${ }^{16}$ Approximately one-half of the patients with the infantile form die from respiratory complications. Longevity studies were not reported in the infantile and childhood forms. Patients affected with adult or odontoHP are believed to have a normal lifespan.

Management Currently, there is no available curative treatment of HP.

(please describe) Therefore, symptomatic treatment in order to improve the clinical features, especially with regard to pain, seizures and other metabolic phenomena (eg, nephrocalcinosis), is of major interest.

Seizures observed in a number of patients with perinatal and infantile HP are pyridoxine responsive and may be therefore treated by using vitamin B6. However, patients may become refractory to this, and in some cases vitamin B6 is not able to control seizures. ${ }^{30-32}$

NSAIDs: the presence of hyperprostaglandinism in childhood HP has been described. 26,33,34 In trials not placebo-controlled, an anti-inflammatory treatment using NSAIDs resulted in a significant improvement of physical activity, a reduction in pain-related complaints and normalisation of prostaglandin (PG) levels. ${ }^{26}$ After drug withdrawal, the positive effect persisted for another 3-6 weeks. NSAID treatment was safe and should be considered in patients with significant complaints about pain. Diet and vitaminotherapy: treatment with zinc and magnesium, the catalytic ions of the enzyme and pyridoxal 5 '-phosphate were reported to have no significant long-term benefit for HP patients. ${ }^{10,35}$ Dietary phosphate restriction is recommended for patients with high serum levels of phosphate. Indeed, patients are often hyperphosphatemic and show nephrocalcinosis.

Furthermore, Pi competitively inhibits TNAP activity and gene expression, which might lower AP activity secondary to the genetic defect. ${ }^{36}$ Especially, for children with persistent growth retardation, an aedequate nutrition with the support of a professional dietician is of major importance. ${ }^{37}$ Supplementation with vitamin D can be recommended according to the guidelines for healthy children. Further, additional vitamin D therapy, as it is used in vitamin D-deficient rickets, should be avoided. An additional supplementation of calcium may result in hypercalcemia and formation of kidney stones.

Physiotherapy: physical exercises like cycling and swimming, as well as physiotherapy, seem to be useful tools for muscle building in order to stabilise the skeleton. Whether a physical workout leads to appositional growth of bone in HP has not been evaluated so far. The maintenance of physical function is of major importance. Especially, in severe forms of disease, trauma to the skeleton must be avoided because resulting fractures take a long time to hea and might be difficult to treat. Surgical as well as conservative therapeutic approaches should be discussed with a HP-experienced clinical centre.

Neurosurgery: premature fusion of cranial sutures may result in secondary ectopia of the cerebellar tonsils (chiari malformation) or formation of hydrosyringomyelia. Neurosurgical interventions are necessary in patients with neurological complications secondary to craniosynostosis, like permanent headache, paralysis or numbness of extremities, as well as papilledema due to elevated intracranial pressure. ${ }^{38}$ With regard to the progressive nature of craniosynostosis, careful surveillance including ophthalmic reassessment, neurological examination and radiographic imaging is recommended throughout childhood until adolescence, and, in presence of Chiari malformation or hydrosyringomyelia, probably throughout lifetime.

-The dentist, especially the paediatric dentist, has a crucial role in the early detection of the milder forms of HP (childhood, adult and odontoHP), as she/he is, most likely, the first health professional to meet the patient and to propose diagnosis hypotheses regarding to the premature loss of primary and, eventually, permanent teeth.

Regular orodental monitoring by a HP-experienced dentist or orthodontist seems to be very important. The emphasis is placed on oral hygiene, appropriate use of fluoride and preventive treatment. Early loss of both primary and/or permanent teeth may be very distressful and may impair maxillary or mandibular development and therefore could be managed through prosthetic appliances.

Prosthetic replacement is helpful for general and linguistic development, as well as social integration of patients in preschool or school or after through adulthood. Possible loosening of remaining teeth should be noted in planning removable dentures. Removable dentures could be considered from 3 years of age.

The successful use of dental implants in adult HP patients has been described in the literature and might be considered as a possible treatment option by the multidisciplinary team of health professionals managing the patient. ${ }^{39}$

3.2 Predictive setting: The tested person is clinically unaffected but carries an increased risk based on family history

(To be answered if in 1.10 'B' was marked)

The age of onset is variable depending on the severity of the disease (for review, see Whyte ${ }^{40}$ ), and the penetrance is not complete, especially in pedigrees where patients are affected with mild forms of HP. Thus, it makes sense to perform genetic testing in relatives of patients. This is interesting for genetic counselling and may help to prevent/delay the onset of clinical symptoms. However, because of the clinical variability and reduced penetrance of some mutations, the predictive power may considerably vary between mutations. The test needs complete information of the tested person about what to expect from the test, and it must be noticed that it may be proposed to relatives of probands only when the familial mutations have been previously characterised.

\subsubsection{Will the result of a genetic test influence lifestyle and prevention?}

If the test result is positive (please describe)

In case of positive test, medical management for prevention of clinical symptoms is required, as described above.

If the test result is negative (please describe)

In case of negative result, the patient has to be reassured and considered as normal with regard to HP.

3.2.2 Which options in view of lifestyle and prevention does a person at-risk have if no genetic test has been done (please describe)?

In general, a person at-risk or with a high probability of being affected by the disease should follow the same recommendations for diagnosis 
and management of potential symptoms and complications, and should be referred to genetic counselling. In general, the clinical suspicion of the disease being present in addition to biochemical testing/confirmation makes the overall diagnosis quite likely.

3.3 Genetic risk assessment in family members of a diseased person (To be answered if in 1.10 ' $\mathrm{C}$ ' was marked)

Severe forms of the disease (perinatal and infantile) are transmitted as an autosomal-recessive trait, whereas both autosomal-recessive and autosomal-dominant transmission have been shown in clinically milder forms. Therefore, the risk of recurrence of severe forms is $25 \%$. In moderate forms, it may be 25\% (recessive transmission), $50 \%$ (dominant transmission) or still different $(<50 \%)$ due to incomplete penetrance in dominant forms. The mutations detected in dominant forms and responsible for moderate $\mathrm{HP}$ are also found in severe recessive HP, associated with other mutations. Thus, siblings of probands with severe HP may develop moderate symptoms, with eventually early loss of teeth but limited if not absent skeletal disease.

\subsubsection{Does the result of a genetic test resolve the genetic situation in that family?}

A negative test may resolve the genetic situation as the disease is very rare, and the tested person/couple have (almost) no risk for having affected children.

A positive test may lead to propose to test the partner, although the couple remain at low risk for severe HP.

\subsubsection{Can a genetic test in the index patient save genetic or other tests in family members?}

In a family with phenotypically comparable affected siblings, a genetic test might not be essential in the yet untested healthy individual.

\subsubsection{Does a positive genetic test result in the index patient enable a predictive test in a family member?}

If a mutation of the index case has been shown to exhibit a dominantnegative effect, a relative carrying this mutation has a risk for developing mild HP (depending on the mutation). However, in this constellation there is still a significant variability in the expectable clinical features.

\subsection{Prenatal diagnosis}

(To be answered if in 1.10 ' $\mathrm{D}$ ' was marked)

For both modes of inheritance, prenatal diagnosis for pregnancies at increased risk is possible if disease-causing mutation(s) of an affected family member is (are) known.

In the prenatal benign form of HP, despite prenatal signs, there may be a spontaneous improvement of skeletal defects. Ultrasounds reveal progressive improvement of the skeletal deformities and mineralisation during the third trimester of the pregnancy. ${ }^{14-16,41}$ The distinction between severe and benign prenatal forms may be difficult and need to combine mutation characterisation and careful ultrasound examination. ${ }^{42}$

\subsubsection{Does a positive genetic test result in the index patient enable a prenatal diagnostic? \\ Yes.}

\section{IF APPLICABLE, FURTHER CONSEQUENCES OF TESTING}

Please assume that the result of a genetic test has no immediate medical consequences. Is there any evidence that a genetic test is nevertheless useful for the patient or his/her relatives? (Please describe)
Genetic testing always has to be founded on genetic counselling. Genetic results with no particular immediate consequence, like in mild autosomal-dominant forms of the disease, still do have significant impact on the long-term decisions in life like partnership, parenthood and long-term surveillance of future medical symptoms. Thus, a test result can be quite useful in the long run, but it also can have stressful implications, especially for the development of a child, and therefore needs to give a complete explanation for parents and psychological support.

\section{CONFLICT OF INTEREST}

The authors declare no conflict of interest.

\section{ACKNOWLEDGEMENTS}

This work was supported by EuroGentest, an EU-FP6 supported NoE, contract number 512148 (EuroGentest Unit 3: 'Clinical genetics, community genetics and public health', Workpackage 3.2). This work was supported by grants from the organisation of patients Hypophosphatasie Europe (EM) and from Alexion company (EM).

1 Zurutuza L, Muller F, Gibrat JF et al: Correlations of genotype and phenotype in hypophosphatasia. Hum Mol Genet 1999; 8: 1039-1046.

2 Muller HL, Yamazaki M, Michigami T et al: Asp361Val Mutant of alkaline phosphatase found in patients with dominantly inherited hypophosphatasia inhibits the activity of the wild-type enzyme. J Clin Endocrinol Metab 2000; 85: 743-747.

3 Lia-Baldini AS, Brun-Heath I, Carrion C et al: A new mechanism of dominance in hypophosphatasia: the mutated protein can disturb the cell localisation of the wildtype protein. Hum Genet 2008; 123: 429-432.

4 Lia-Baldini AS, Muller F, Taillandier A et al: A molecular approach to dominance in hypophosphatasia. Hum Genet 2001; 109: 99-108.

5 Fauvert D, Brun-Heath I, Lia-Baldini AS et al: Mild forms of hypophosphatasia mostly result from dominant negative effect of severe alleles or from compound heterozygosity for severe and moderate alleles. BMC Med Genet 2009; 10: 51.

6 Whyte MP, Teitelbaum SL, Murphy WA, Bergfeld MA, Avioli LV: Adult hypophosphatasia. Clinical, laboratory, and genetic investigation of a large kindred with review of the literature. Medicine (Baltimore) 1979; 58: 329-347.

7 Eberle F, Hartenfels S, Pralle H, Kabisch A: Adult hypophosphatasia without apparent skeletal disease: 'odontohypophosphatasia' in four heterozygote members of a family. Klin Wochenschr 1984; 62: 371-376.

8 Eastman JR, Bixler D: Clinical, laboratory, and genetic investigations of hypophosphatasia: support for autosomal dominant inheritance with homozygous lethality. J Craniofac Genet Dev Biol 1983; 3: 213-234.

9 Spentchian M, Brun-Heath I, Taillandier A et al: Characterisation of missense mutations and large deletions in the ALPL gene by sequencing and quantitative multiplex PCR of short fragments. Genet Test 2006; 10: 252-257.

10 Fraser D: Hypophosphatasia. Am J Med 1957; 22: 730-746.

11 Mornet E, Yvard A, Taillandier A, Fauvert D, Simon-Bouy B: A molecular-based estimation of the prevalence of hypophosphatasia in the European population. Ann Hum Genet 2011; 75: 439-445.

12 Greenberg CR, Evans JA, McKendry-Smith S et al: Infantile hypophosphatasia: localisation within chromosome region 1p36.1-34 and prenatal diagnosis using linked DNA markers. Am J Hum Genet 1990; 46: 286-292.

13 Whyte MP, Essmyer K, Geimer M, Mumm S: Homozygosity for TNSALP mutation $1348 \mathrm{c}>\mathrm{T}$ (Arg433Cys) causes infantile hypophosphatasia manifesting transient disease correction and variably lethal outcome in a kindred of black ancestry. J Pediatr 2006; 148: 753-758.

14 Pauli RM, Modaff P, Sipes SL, Whyte MP: Mild hypophosphatasia mimicking severe osteogenesis imperfecta in utero: bent but not broken. Am J Med Genet 1999; 86: 434-438.

15 Moore CA, Curry CJ, Henthorn PS et al: Mild autosomal dominant hypophosphatasia: in utero presentation in two families. Am J Med Genet 1999; 86: 410-415.

16 Wenkert D, McAlister WH, Coburn SP et al: Hypophosphatasia: nonlethal disease despite skeletal presentation in utero (17 new cases and literature review). J Bone Miner Res 2011; 26: 2389-2398.

17 Reibel A, Maniere MC, Clauss F et al: Orodental phenotype and genotype findings in all subtypes of hypophosphatasia. Orphanet J Rare Dis 2009; 4: 6 .

18 Whyte MP, Wenkert D, McAlister WH et al: Chronic recurrent multifocal osteomyelitis mimicked in childhood hypophosphatasia. J Bone Miner Res 2009; 24: 1493-1505.

19 Deeb AA, Bruce SN, Morris AA, Cheetham TD: Infantile hypophosphatasia: disappointing results of treatment. Acta Paediatr 2000; 89: 730-733.

20 Sutton RA, Mumm S, Coburn SP, Ericson KL, Whyte MP: 'Atypical femoral fractures' during bisphosphonate exposure in adult hypophosphatasia. J Bone Miner Res 2012; 27: 987-994. 
21 Whyte MP, Mumm S, Deal C: Adult hypophosphatasia treated with teriparatide. J Clin Endocrinol Metab 2007; 92: 1203-1208.

22 Camacho PM, Painter S, Kadanoff R: Treatment of adult hypophosphatasia with teriparatide. Endocr Pract 2008; 14: 204-208.

23 Gagnon C, Sims NA, Mumm S et al: Lack of sustained response to teriparatide in a patient with adult hypophosphatasia. J Clin Endocrinol Metab 2010; 95: 1007-1012.

24 Schalin-Jantti C, Mornet E, Lamminen A, Valimaki MJ: Parathyroid hormone treatment improves pain and fracture healing in adult hypophosphatasia. J Clin Endocrinol Metab 2010; 95: 5174-5179.

25 Girschick HJ, Seyberth HW, Huppertz HI: Treatment of childhood hypophosphatasia with nonsteroidal antiinflammatory drugs. Bone 1999; 25: 603-607.

26 Girschick HJ, Schneider P, Haubitz I et al: Effective NSAID treatment indicates that hyperprostaglandinism is affecting the clinical severity of childhood hypophosphatasia. Orphanet J Rare Dis 2006; 1: 24.

27 Millan JL, Narisawa S, Lemire I et al: Enzyme replacement therapy for murine hypophosphatasia. J Bone Miner Res 2008; 23: 777-787.

28 Whyte MP, Greenberg CR, Salman NJ et al: Enzyme-replacement therapy in lifethreatening hypophosphatasia. N Engl J Med 2012; 366: 904-913.

29 Sugano H, Matsumoto T, Miyake K et al: Successful gene therapy in utero for lethal murine hypophosphatasia. Hum Gene Ther 2012; 23: 399-406.

30 Baumgartner-Sigl S, Haberlandt E, Mumm S et al: Pyridoxine-responsive seizures as the first symptom of infantile hypophosphatasia caused by two novel missense mutations (c.677T > C, p.M226T; c.1112C > T, p.T371I) of the tissue-nonspecific alkaline phosphatase gene. Bone 2007; 40: 1655-1661.

31 Balasubramaniam S, Bowling F, Carpenter $\mathrm{K}$ et al: Perinatal hypophosphatasia presenting as neonatal epileptic encephalopathy with abnormal neurotransmitte metabolism secondary to reduced co-factor pyridoxal-5'-phosphate availability. $J$ Inherit Metab Dis 2010; doi:10.1007/s10545-009-9012-y.
32 Hofmann C, Liese J, Schwarz T et al: Compound heterozygosity of two functional null mutations in the ALPL gene associated with deleterious neurological outcome in an infant with hypophosphatasia. Bone 2013; 55: 150-157.

33 Girschick HJ, Haubitz I, Hiort O, Schneider P: Long-term follow-up of bone mineral density in childhood hypophosphatasia. Joint Bone Spine 2007; 74: 263-269.

34 Girschick HJ, Mornet E, Beer M, Warmuth-Metz M, Schneider P: Chronic multifocal non-bacterial osteomyelitis in hypophosphatasia mimicking malignancy. BMC Pediatr 2007; 7: 3

35 Wolfish NM, Heick H: Hyperparathyroidism and infantile hypophosphatasia: effect of prednisone and vitamin K therapy. J Pediatr 1979; 95: 1079-1081.

36 Whyte MP: Physiological role of alkaline phosphatase explored in hypophosphatasia. Ann N Y Acad Sci 2010; 1192: 190-200.

37 Moulin P, Vaysse F, Bieth E et al: Hypophosphatasia may lead to bone fragility: don't miss it. Eur J Pediatr 2009; 168: 783-788.

38 Collmann H, Mornet E, Gattenlohner S, Beck C, Girschick H: Neurosurgical aspects of childhood hypophosphatasia. Childs Nerv Syst 2009; 25: 217-223.

39 Lynch CD, Ziada HM, Buckley LA, O'Sullivan VR, Aherne T, Aherne S: Prosthodontic rehabilitation of hypophosphatasia using dental implants: a review of the literature and two case reports. J Oral Rehabil 2009; 36: 462-468.

40 Whyte MP: Hypophosphatasia and the role of alkaline phosphatase in skeletal mineralization. Endocr Rev 1994; 15: 439-461.

41 Stevenson DA, Carey JC, Coburn SP et al: Autosomal recessive hypophosphatasia manifesting in utero with long bone deformity but showing spontaneous postnata improvement. J Clin Endocrinol Metab 2008; 93: 3443-3448.

42 Sinico M, Levaillant JM, Vergnaud A et al: Specific osseous spurs in a lethal form of hypophosphatasia correlated with 3D prenatal ultrasonographic images. Prenat Diagn 2007; 27: 222-227. 